\title{
A NOTE ON THE ENTROPY OF SKEW PRODUCT TRANSFORMATIONS ${ }^{1}$
}

ROY L. ADLER

Let $(X, X, \lambda)$ and $(Y, Y, \mu)$ be two Lebesgue spaces with $X$ and $Y$ the fields of measurable subsets of $X$ and $Y$ respectively. $\lambda$ and $\mu$ are countably additive measures on $x$ and $Y$ respectively with $\lambda(X)$ $=\mu(Y)=1$. Let $(Z, Z, \nu)=(X \times Y, x \times Y, \lambda \times \mu)$ denote the direct product of the above measure spaces. Let $\phi$ be a measure preserving transformation on $X$, and for each $x \in X$ let $\psi_{x}$ be measure preserving transformation on $Y$. If the family $\left\{\psi_{x}: x \in X\right\}$ of measure preserving transformations satisfies certain measurability conditions (see $[2, \mathrm{pp}$. $83,84]$ ), then it can be shown that the transformation $T$ defined by

$$
T(x, y)=\left(\phi x, \psi_{x} y\right)
$$

is a measure preserving transformation on $Z . T$ is called the skew product transformation of $\phi$ with the family $\left\{\psi_{x}: x \in X\right\}$.

The purpose of this work is to compute the entropy $h(T)$. (For definition of entropy of a measure preserving transformation and the associated notation consult [3] and [4].) The natural conjecture is

$$
h(T)=h(\phi)+\int_{X} h\left(\psi_{x}\right) \lambda(d x) .
$$

This conjecture is substantiated in several instances. When $\psi_{x}=\psi$ for all $x \in X,\left(^{*}\right)$ reduces to the formula for direct product transformations (see [4] formula $(\beta)$ ); i.e.,

$$
h(T)=h(\phi)+h(\psi) .
$$

For $\phi=I$ the identity transformation on $X,\left(^{*}\right)$ reduces to the case of decomposition of a measure preserving transformation into components (see [4] formula $(\epsilon)$ ); i.e.,

$$
h(T)=\int_{\mathbf{X}} h\left(\psi_{x}\right) \lambda(d x) .
$$

When $Y=$ unit interval and $\psi_{x} y=y+\alpha(x)(\bmod 1)$ where $\alpha(\cdot)$ is some real-valued measurable function on $X$, Abramov [1] has shown

$$
h(T)=h(\phi)
$$

Received by the editors May 2, 1962.

1 The material in this paper was included in the author's doctoral dissertation submitted to Yale University (1961) under the direction of Professor S. Kakutani. 
which is again a special case of $\left(^{*}\right)$ since $h\left(\psi_{x}\right)=0, x \in X$.

In general $\left(^{*}\right)$ is not true. However, we shall derive a formula which differs from $\left(^{*}\right)$ in the function occurring within the integral.

Let $x_{k}, k=1,2, \cdots$ be an increasing sequence of finite subfields of $X$ whose union generates $X$ and let $Z_{m}, m=1,2, \cdots$ be an increasing sequence of finite subfields of $\mathcal{Z}$ whose union generates $\mathcal{Z}$. Let ${ }_{T}^{n} \mathbf{Z}_{m}$ denote $\boldsymbol{Z}_{m} \bigvee T Z_{m} \bigvee \cdots \vee T^{n-1} \mathcal{Z}_{m}$. Denote by $\left(\mathcal{Z}_{m}\right)_{x}$ the field of subsets of $Y$ which consists of $x$-sections of sets in $Z_{m}$. We observe

$$
\begin{aligned}
\left({ }_{T}^{n} Z_{m}\right)_{x}= & \left(Z_{m}\right)_{x} \vee \psi_{\phi^{-1} x}\left(Z_{m}\right)_{\phi^{-1} x} \vee \cdots \\
& \vee \psi_{\phi^{-1} x} \psi_{\phi^{-2} x} \cdots \psi_{\phi^{-n+1} x}\left(Z_{m}\right)_{\phi^{-n+1} x}
\end{aligned}
$$

We shall employ an ambiguity whose meaning will be clear in context by having the symbols $x_{k}$ represent either fields of measurable subsets of $X$ or fields of cylinder sets in $Z$ based on subsets of $X$ in $\mathfrak{X}_{k}$. Keeping this ambiguity in mind consider the following relation between mean entropy and mean (conditional) entropy of finite fields (see $[4$, p. 980])

$$
\begin{aligned}
H\left({ }_{T}^{n} X_{k} \bigvee{ }_{T}^{n} Z_{m}\right) & =H\left({ }_{T}^{n} X_{k}\right)+H\left({ }_{T}^{n} Z_{m} \mid{ }_{T}^{n} X_{k}\right) \\
& =H\left({ }_{\phi}^{n} X_{k}\right)+H\left({ }_{T}^{n} Z_{m} \mid{ }_{T}^{n} X_{k}\right) .
\end{aligned}
$$

Dividing by $n$ and observing ${ }_{T}^{n} x_{k} \subseteq X$ we have the inequality

$$
\frac{H\left({ }_{T}^{n} X_{k} \vee{ }_{T}^{n} \mathcal{Z}_{m}\right)}{n} \geqq \frac{H\left({ }_{\phi}^{n} X_{k}\right)}{n}+\frac{H\left({ }_{T}^{n} \mathcal{Z}_{m} \mid X\right)}{n} .
$$

The definition of mean (conditional) entropy yields

$$
H\left({ }_{r}^{n} \mathrm{Z}_{m} \mid x\right)=\int_{X} H\left(\left({ }_{T}^{n} \mathrm{Z}_{m}\right)_{x}\right) \lambda(d x) .
$$

By replacing the function in the integral with (1) and substituting in

(2) we get

$$
\begin{aligned}
& \frac{H\left({ }_{T}^{n} \mathfrak{X}_{k} \vee{ }_{r}^{n} \mathcal{Z}_{m}\right)}{n} \geqq \frac{H\left({ }_{\phi}^{n} X_{k}\right)}{n} \\
& +\int_{X} \frac{H\left(\left(\mathcal{Z}_{m}\right)_{x} \bigvee \psi_{\phi^{-1} x}\left(\mathcal{Z}_{m}\right)_{\phi^{1-x}} \bigvee \cdots \vee \psi_{\phi^{-1} x} \psi_{\phi^{-2} x} \cdots \psi_{\phi^{-n+1} x}\left(\mathcal{Z}_{m}\right)_{\phi^{-n+1} x}\right)}{n} \\
& \quad \cdot \lambda(d x)
\end{aligned}
$$

The following identity can be established (see for instance $[3$, p. 33]): 


$$
\begin{aligned}
& H\left(\left(Z_{m}\right)_{x} \bigvee \psi_{\phi^{-1} x}\left(Z_{m}\right)_{\phi^{-1} x} \bigvee \cdots \vee \psi_{\phi^{-1} x} \psi_{\phi^{-2} x} \cdots \psi_{\phi^{-n+1} x}\left(Z_{m}\right)_{\phi^{-n+1} x}\right) \\
&=\sum_{j=0}^{n-1} H\left(\left(Z_{m}\right)_{\phi^{-} j_{x}} \mid \psi_{\phi^{-i-1} x}\left(Z_{m}\right)_{\phi^{-i-1} x} \bigvee\right. \cdots \\
&\left.\vee \psi_{\phi^{-j-1} x} \cdots \psi_{\phi^{-n+1} x}\left(Z_{m}\right)_{\phi^{-n+1} x}\right)
\end{aligned}
$$

As $n$ tends to $\infty$,

$$
H\left(\left(Z_{m}\right)_{\phi^{-i} x} \mid \psi_{\phi^{-i-1} x}\left(Z_{m}\right)_{\phi^{-i-1} x} \bigvee \cdots \vee \psi_{\phi^{-j-1} x} \cdots \psi_{\phi^{-n+1} x}\left(Z_{m}\right)_{\phi^{-n+1} x}\right)
$$

decreases to a limit which we denote by $f_{\phi}\left(x, Z_{m}, j\right)$. Likewise, as $n \rightarrow \infty$,

$$
\begin{aligned}
& \int_{X} H\left(\left(Z_{m}\right)_{\phi^{-i} x} \mid \psi_{\phi^{-i-1} x}\left(\mathcal{Z}_{m}\right)_{\phi^{-i-1} x} \vee \cdots\right. \\
& \left.\bigvee \psi_{\phi^{-j-1} x} \cdots \psi_{\phi^{-n+1} x}\left(Z_{m}\right)_{\phi^{-n+1} x}\right) \lambda(d x)
\end{aligned}
$$

tends to

$$
\int_{X} f_{\phi}\left(x, Z_{m}, j\right) \lambda(d x)
$$

By virtue of the fact that $\phi$ is measure preserving it follows that

$$
\int_{X} f_{\phi}\left(x, \mathbf{Z}_{m}, j\right) \lambda(d x)=\int_{X} f_{\phi}\left(x, \mathbf{Z}_{m}, 0\right) \lambda(d x) .
$$

Having outgrown the need for a function of three variables we replace $f_{\phi}\left(x, Z_{m}, 0\right)$ by simply $f_{\phi}\left(x, Z_{m}\right)$. From (4) and (5) and the fact that ordinary convergence implies Cesaro convergence we obtain

$$
\int_{X} \frac{H\left(\left({ }^{n} \mathcal{Z}_{m}\right)_{x}\right)}{n} \lambda(d x) \rightarrow \int_{X} f_{\phi}\left(x, Z_{m}\right) \lambda(d x)
$$

as $n \rightarrow \infty$. Taking limits in (3) as $n \rightarrow \infty$ yields

$$
h(T) \geqq h\left(T, x_{k} \vee \mathcal{Z}_{m}\right) \geqq h\left(\phi, x_{k}\right)+\int_{X} f_{\phi}\left(x, \mathbf{Z}_{m}\right) \lambda(d x) .
$$

Then letting $k \rightarrow \infty$ we get

$$
h(T) \geqq h(\phi)+\int_{X} f\left(x, \mathbf{Z}_{m}\right) \lambda(d x) .
$$

In order to obtain the reverse inequality consider 
(9)

$$
\frac{H\left({ }_{r}^{n l} Z_{m}\right)}{n l} \leqq \frac{H\left({ }_{r}^{n l} X_{k} \vee{ }_{r}^{n l} Z_{m}\right)}{n l}=\frac{H\left({ }_{r}^{n l} X_{k}\right)}{n l}+\frac{B\left({ }_{r}^{n l} Z_{m} \mid{ }_{r}^{n l} X_{k}\right)}{n l}
$$

$$
\leqq \frac{B\left({ }_{\phi}^{n l} X_{x}\right)}{n l}+\frac{B\left({ }_{T}^{n l} \mathrm{Z}_{m} \mid{ }_{T}^{n l} X_{k}\right)}{n l} .
$$

Now

$$
\begin{aligned}
H\left({ }_{r}^{n l} Z_{m} \mid{ }_{r}^{n l} X_{k}\right) & \left.\leqq \sum_{i=0}^{n-1} H\left(T^{i l}{ }_{r}^{l} Z_{m}\right) \mid{ }_{T}^{n l} X_{k}\right) \leqq \sum_{i=0}^{n-1} H\left(T^{i l}\left({ }_{r}^{l} Z_{m}\right) \mid T^{i l} x_{k}\right) \\
& \leqq n H\left({ }_{r}^{l} Z_{m} \mid X_{k}\right) .
\end{aligned}
$$

Combining (9) and (10) we have

$$
\frac{\left(B_{T}^{n l} Z_{m}\right)}{n l} \leqq \frac{B\left({ }_{\phi}^{n l} X_{k}\right)}{n l}+\frac{B\left({ }_{r}^{l} Z_{m} \mid X_{k}\right)}{l} .
$$

Letting $n \rightarrow \infty$ in (11)

$$
h\left(T, \mathrm{Z}_{m}\right) \leqq h\left(\phi, \mathfrak{X}_{k}\right)+\frac{H\left({ }_{r}^{l} \mathrm{Z}_{m} \mid \mathfrak{X}_{k}\right)}{l},
$$

and letting $k \rightarrow \infty$ in (12)

$$
h\left(T, \mathrm{Z}_{m}\right) \leqq h(\phi)+\frac{H\left({ }_{r}^{l} \mathrm{Z}_{m} \mid x\right)}{l},
$$

or equivalently,

$$
h\left(T, \mathrm{Z}_{m}\right) \leqq h(\phi)+\int_{X} \frac{\left.B\left({ }_{r}^{l} \mathrm{Z}_{m}\right)_{x}\right)}{l} \lambda(d x) .
$$

Next letting $l \rightarrow \infty$ and combining the result with (8) we have

$$
h\left(T, \mathbf{Z}_{m}\right) \leqq h(\phi)+\int_{X} f_{\phi}\left(x, \mathbf{Z}_{m}\right) \lambda(d x) \leqq h(T) .
$$

Now

$$
f_{\phi}\left(x, Z_{m}\right)=\lim _{n \rightarrow \infty} \frac{B\left(\left({ }_{r}^{n} Z_{m}\right)_{x}\right)}{n},
$$

and it is clear that $f\left(x, Z_{m}\right)$ increases with $m$ to a possibly infinite but 
well defined limit $f_{\phi}(x)$. Since $\lim _{m \rightarrow \infty} h\left(T, Z_{m}\right)=h(T)$, it follows from (14) that

$$
h(T)=h(\phi)+\int_{X} f_{\phi}(x) \lambda(d x)
$$

Of course we would like to establish

$$
\int_{X} f_{\phi}(x) \lambda(d x)=\int_{X} h\left(\psi_{x}\right) \lambda(d x)
$$

where

$$
\begin{aligned}
f_{\phi}(x) & =\lim _{m \rightarrow \infty} f_{\phi}\left(x, Z_{m}\right) \\
f_{\phi}\left(x, Z_{m}\right) & =\lim _{n \rightarrow \infty} \frac{H\left(\left(Z_{m}\right)_{x} \bigvee \psi_{\phi^{-1} x}\left(Z_{m}\right)_{\phi^{-1} x} \vee \cdots \vee \psi_{\phi^{-1} x} \psi_{\phi^{-2} x} \cdots\right.}{\left.\psi_{\phi^{-n+1} x}\left(\mathcal{Z}_{m}\right)_{\phi^{-n+1} x}\right)} \\
h\left(\psi_{x}\right) & =\lim _{m \rightarrow \infty} h\left(\psi_{x}, Z_{m}\right) \\
h\left(\psi_{x}, Z_{m}\right) & =\lim _{n \rightarrow \infty} \frac{H\left(\left(Z_{m}\right)_{x} \bigvee \psi_{x}\left(Z_{m}\right)_{x} \vee \cdots \vee \psi_{x}^{n-1}\left(Z_{m}\right)_{x}\right)}{n} .
\end{aligned}
$$

The quantities $h\left(\psi_{x}, \mathbf{Z}_{m}\right)$ and $f\left(x, \mathbf{Z}_{m}\right)$ are different by the nature of their definitions. Perhaps only mild restrictions are required so that the differences can be eliminated by integration to yield $(* * *)$. The following example, however, reveals that in general $(* * *)$ is not true: let $X=X_{1} \cup X_{2}$ where $m\left(X_{1}\right)=m\left(X_{2}\right)=\frac{1}{2}$; let $\psi_{x}=\psi, x \in X_{1}$ and $\psi_{x}=\psi^{-1}, x \in X_{2}$ where $\psi$ is a measure preserving transformation on $Y$ such that $h(\psi) \neq 0$; and let $\phi$ be a measure preserving transformation on $X$ such that $\phi X_{1}=X_{2}, \phi X_{2}=X_{1}$ and $\phi^{2}=I$. Then for $T:(x, y)$ $\rightarrow\left(\phi x, \psi_{x} y\right)$ we have $T^{2}$ is the identity transformation on $X \times Y$ so that $h(T)=0$; but $h(\phi)+\int_{x} h\left(\psi_{x}\right) \lambda(d x)=h(\psi) \neq 0$.

\section{REFERENCES}

1. L. M. Abramov, On entropy of flows, Dokl. Akad. Nauk SSSR 128 (1959), 873875.

2. H. Anzai, Ergodic skew product transformations on the torus, Osaka Math. J. 3 (1959), 83-99.

3. P. R. Halmos, Entropy in ergodic theory, Univ. of Chicago, 1959. (Mimeographed Notes)

4. V. A. Rokhlin, Entropy of metric automorphism, Dokl. Akad. Nauk SSSR 124 (1959), 768-771.

International Business Machines Corporation 